\title{
Stochastic chemical enrichment in the early Galaxy
}

\author{
Torgny Karlsson ${ }^{1}$ and Bengt Gustafsson ${ }^{2}$ \\ ${ }^{1}$ NORDITA, Blegdamsvej 17, DK-2100 Copenhagen $\varnothing$, Denmark \\ email: Karlsson@nordita.dk \\ ${ }^{2}$ Department of Astronomy and Space Physics, Box 515, SE-75120 Uppsala, Sweden \\ email: Bengt.Gustafsson@astro.uu.se
}

\begin{abstract}
A stochastic model of the chemical enrichment of metal-poor systems by core-collapse supernovae is used to study the scatter in relative elemental abundances in extremely metalpoor stars. The resulting scatter in abundance ratios is demonstrated to be crucially dependent on the as yet uncertain supernovae yields. The relatively small star-to-star scatter observed in many of these abundance ratios, e.g. by Cayrel et al. (2004), is tentatively explained by the averaging of a large number of contributing supernovae and by the cosmic selection effects favoring contributions from supernovae in a certain mass range for the most metal-poor stars. "Spurs", very narrow sequences in abundance-ratio diagrams, may disclose a single-supernova origin of the elements of the stars on the sequence and would thus be an indication of an unmixed interstellar medium (ISM). Verification of the existence of such features, called single supernova sequences (SSSs), is challenging. This will require samples of several hundred stars with abundance ratios observed to accuracies of 0.05 dex or better.
\end{abstract}

Keywords. Nuclear reactions, nucleosynthesis - stars: abundances - stars: Population II stars: supernovae: general - ISM: bubbles - ISM: evolution - Galaxy: evolution - Galaxy: halo galaxies: evolution

\section{Introduction}

One decade ago, the first major attempt to determine elemental abundances in a large sample of extremely metal-poor $([\mathrm{Fe} / \mathrm{H}]<-2.5)$ stars revealed a significant star-to-star scatter in many abundance ratios (McWilliam et al. 1995). This scatter was interpreted as an indication of a chemically inhomogeneous interstellar medium (ISM) at the time of formation of these stars. As pointed out by Audouze \& Silk (1995), large chemical fluctuations in the ISM would naturally have developed during the first star formation episode since, at that time, only a small number of supernovae ( $\mathrm{SNe}$ ) would have been able to contribute to the local abundance of elements.

In contrast to this picture, a number of recent observational studies (Arnone et al. 2005; Barklem et al. 2005; Cayrel et al. 2004; Carretta et al. 2002; Nissen et al. 1994) have shown that the star-to-star scatter in the extremely metal-poor regime tend to be much smaller than previously anticipated, also for the population of stars with metallicity as low as $[\mathrm{Fe} / \mathrm{H}]=-4$. The conclusion drawn from this is thus the opposite; namely that the ISM was homogeneous and well-mixed, even in the early Galaxy. One should remember, however, that the large scatter persists for some elements, such as for carbon and the neutron-capture elements. In fact, the exclusion of bona fide carbon-enhanced, metal-poor stars (with $[\mathrm{C} / \mathrm{Fe}]>1$ ) from the population of 'normal' stars could lead to biased and inaccurate conclusions as regards the early evolution of the Milky Way Galaxy. 

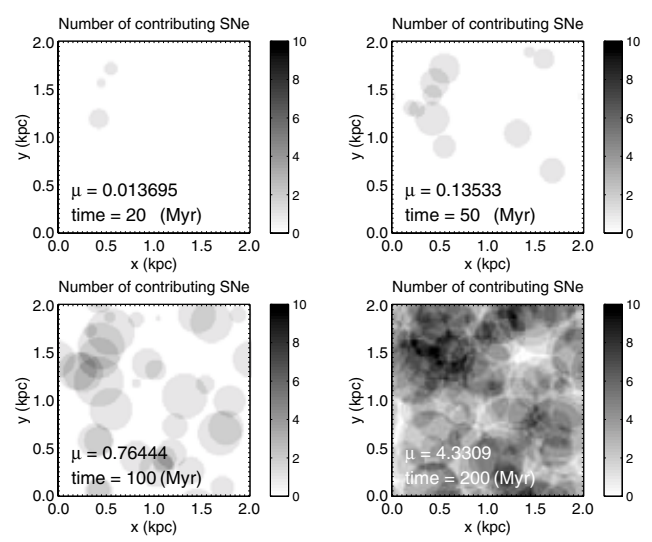

Figure 1. The continuous enrichment of chemical elements in the ISM. In the beginning, only a few exploding stars have enriched their immediate surroundings in freshly synthesized elements. At later times more regions are enriched. In addition, the older regions (i.e., mixing volumes) have expanded due to the large-scale mixing. The parameter $\mu=\mu(t)$, defined in Eq. (2.3), denotes the average number of $\mathrm{SNe}$ that have enriched a random volume element in space at time $t$. The shaded bars indicate the number of contributing SNe.

Naively, one would expect that a small scatter can only be obtained if stars were formed out of gas enriched by many SNe. Does this mean that averaging effects were already in play at $[\mathrm{Fe} / \mathrm{H}]=-4$ ? Several chemical evolution models developed to study the effect of small number statistics on the build-up of chemical elements in the early Galaxy do predict a large scatter at these metallicities (e.g., Travaglio et al. 2001; Argast et al. 2000; Tsujimoto et al. 1999). However, these models do not take into account the continuous, large-scale mixing of the ISM driven, e.g., by turbulent diffusion and cloudcloud collisions. We have developed a new chemical evolution model in order to study the effect of such an extra mixing (Karlsson 2005, hereafter Paper I). This model also allows for (space-independent) infall of pristine gas.

In Sect. 2 the stochastic chemical enrichment model is outlined, including a definition of the mixing volume picture. The results are presented in Sect. 3 and the conclusions are given in Sect. 4. As we will see, a small star-to-star scatter does not necessarily imply a well-mixed ISM (Karlsson \& Gustafsson 2005, hereafter Paper II).

\section{The model}

At some point in the past, the ISM must have experienced a first episode of star formation and from that point and onward, gradually changed from an inhomogeneous, unmixed state of chemical enrichment into a homogeneous and more mixed state. This homogenization is driven by two effects (Fig. 1): the continuous increase of the number of exploding stars and the continuous mixing of interstellar gas, e.g., by turbulent motions. This mixing, however, is not instantaneous.

\subsection{The mixing volume picture}

A key concept in our model of early enrichment of the ISM is the mixing volume $V_{\text {mix }}=V_{\text {mix }}(t)$. One mixing volume is assumed to be generated by each $\mathrm{SN}$ and is defined as the total volume enriched by debris from this SN, including the region directly affected by the explosion as well as those regions which are reached due to turbulent 
motions and cloud-cloud collisions in the ISM. Ascribing these motions to different random walk processes, where the rms distance $R_{\mathrm{rms}}$ increases as $t^{1 / 2}$ (Bateman \& Larson 1993), and neglecting the relatively small initial expansion of the SN remnant itself, we estimate that the mixing volume should on average increase as

$$
V_{\text {mix }}(t)=\frac{4 \pi}{3} R_{\mathrm{rms}}^{3}=\frac{4 \pi}{3}\left(\sigma_{\mathrm{mix}} t\right)^{3 / 2} .
$$

Here $t$ denotes the time after the SN explosion and $\sigma_{\text {mix }}$ is a combined cross-sectional expansion rate for the processes responsible for the bulk distribution of heavy elements. From the data given in Bateman \& Larson (1993) we find $\sigma_{\text {mix }}=7 \times 10^{-4} \mathrm{kpc}^{2} \mathrm{Myr}^{-1}$.

As time goes on, the mixing volumes begin to overlap in space (see Fig. 1). Chemical inhomogeneities are developed by subsequent generations of SNe that explode within earlier generations of mixing volumes and enrich the gas further. At any instant in time, different regions of the ISM will be enriched by different numbers of SNe and there exists a distribution $w_{\mathrm{ISM}}(k, t)$ describing the probability of finding $k$ overlapping mixing volumes at time $t$ somewhere in the ISM. Via this distribution, we are able to quantify and map the abundance distributions in subsequently formed stars.

\subsection{A statistical description of chemical inhomogeneities in the ISM}

The form of $w_{\text {ISM }}$ may be inferred from the spatial Poisson process (see Paper II for discussion). Assuming that the $\mathrm{SNe}$ are randomly distributed over space with an average rate $u_{\mathrm{SN}}\left(=0.25 \mathrm{kpc}^{-3} \mathrm{Myr}^{-1}\right.$, Paper II), we may set the probability of finding a region enriched by $k$ SNe to be

$$
w_{\text {ISM }}(k, t)=P o(k, \mu(t))=e^{-\mu(t)} \mu(t)^{k} / k !,
$$

where $\mu(t)$ denotes the integrated volume affected by SNe expressed in units of the total volume of the system. If, at time $t, V_{\text {mix }}\left(t-t^{\prime}\right)$ is the size of the volumes enriched by material from SNe that exploded at time $t^{\prime}$ then

$$
\mu(t)=\int_{0}^{t} V_{\text {mix }}\left(t-t^{\prime}\right) u_{\mathrm{SN}}\left(t^{\prime}\right) \mathrm{d} t^{\prime} .
$$

With this definition, $\mu(t)$ is a measure of the average number of SNe that have enriched a random volume element in space at time $t$. The chemical inhomogeneities should be well described by Poisson statistics as long as the SNe are assumed to be randomly distributed in the ISM and $\mu \ll N_{\mathrm{SN}}$, the total number of SNe in the system.

From Eqs. (2.1)-(2.3) we may now derive analytical expressions for the distribution $f_{M_{k}}$ of mixing masses and the number of persisting stars formed by gas polluted by $k$ different supernovae, $n_{k}$, with $k$ ranging from zero to high numbers (see Paper I). The mixing mass is the total amount of gas within the mixing volume $V_{\text {mix }}$, in which the newly synthesized elements are mixed. For the mixing masses, we find rather narrow distributions extending from about $10^{5}$ to $10^{6} \mathcal{M}_{\odot}$ for $k=1$, assuming a gas density of $n=0.1 \mathrm{~cm}^{-3}$. The widths of the distributions for $k=100$ are on the order of $5-10$ times larger, depending on the input parameters (see Paper I, Fig. 3). Taking the high-mass initial mass function (IMF) to be of Salpeter form, with the yields for each SN initial mass, we may now also for each $k$ calculate the expected distribution of stellar abundances of any chemical element, $A$. A summation over $k$ leads to the final distributions of stars with different abundances and abundance ratios (Paper I). 

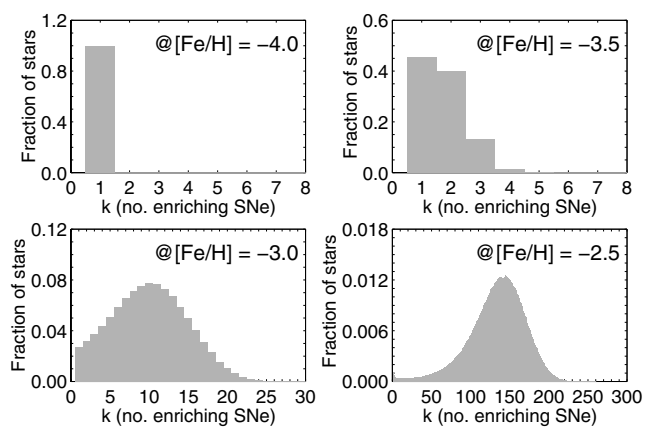

Figure 2. The fraction of low-mass stars enriched by $k$ SNe at a given metallicity. The increase of the average number of contributing $\mathrm{SNe}$ is substantial over the metallicity range $-4 \leqslant[\mathrm{Fe} / \mathrm{H}] \leqslant-2.5$.

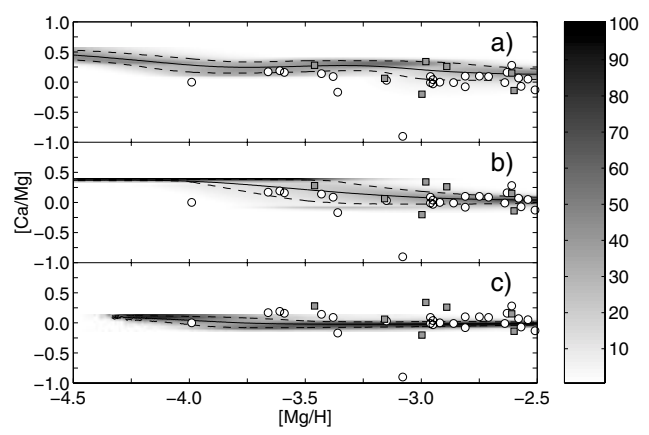

Figure 3. The predicted distributions of stars in the $[\mathrm{Ca} / \mathrm{Mg}]-[\mathrm{Mg} / \mathrm{H}]$ plane renormalized to equal number per $[\mathrm{Mg} / \mathrm{H}]$-bin. a) Yields by Woosley \& Weaver (1995). b) Yields by Umeda \& Nomoto (2002). c) Yields by Chieffi \& Limongi (2004). The open circles denote observations of giants by Cayrel et al. (2004) and the gray squares denote observations of dwarfs by Cohen et al. (2004). The shaded bar indicates the relative density of stars (arb. units).

\section{Results}

We have adopted three different sets of stellar yields calculated from initially metal-free star models: the ones by Woosley \& Weaver (1995), Umeda \& Nomoto (2002), and Chieffi \& Limongi (2004). The remaining input parameters (i.e., $n, u_{\mathrm{SN}}, \sigma_{\mathrm{mix}}$, and IMF) are inferred from different studies in the literature and are not optimized to fit the observations. Before discussing some different abundance ratio diagrams, let us first comment on the metallicity distribution of the population of extremely metal-poor Galactic halo stars.

\subsection{The metallicity distribution}

The predicted number of Galactic halo stars decreases roughly exponentially with decreasing metallicity (i.e., $[\mathrm{Fe} / \mathrm{H}]$ ). Stars only enriched by a single SN are mostly distributed around $[\mathrm{Fe} / \mathrm{H}]=-4$, although approximately $1 \%$ may have a metallicity as high as $[\mathrm{Fe} / \mathrm{H}]=-2.5$. Due to the non-instantaneous mixing, there is a sharp drop in number of stars below $[\mathrm{Fe} / \mathrm{H}]=-4$. On the other hand, our model predicts a higher number of SNe contributing to the chemical enrichment at a given metallicity, e.g., at $[\mathrm{Fe} / \mathrm{H}]=-2.5$, as compared to models neglecting the large-scale mixing. Fig. 2 shows the fractions of Galactic halo stars enriched by $k$ SNe at four different metallicities.

\subsection{Stellar abundance distributions}

Fig. 3 shows the evolution of $[\mathrm{Ca} / \mathrm{Mg}]$ as a function of $\mathrm{Mg}$ abundance. In general, there is a good agreement between observations and predictions for all three sets of stellar yields. In Fig. 3a, the predicted star-to-star scatter in $[\mathrm{Ca} / \mathrm{Mg}]$ is nearly constant over the entire range in metallicity, in accordance with observations, despite the fact that stars with the lowest $\mathrm{Mg}$ abundances are mostly enriched by $1-2 \mathrm{SNe}$ while stars at $[\mathrm{Mg} / \mathrm{H}]=-2.5$ are on average enriched by more than $100 \mathrm{SNe}$ (cf. Fig. 2). In Fig. 3b, the situation is even more extreme. Here the simulated scatter reaches a maximum level around $[\mathrm{Mg} / \mathrm{H}]=$ -3.4 and then decreases again towards lower metallicities. This phenomenon is explained by a combination of two effects: the averaging of a large number of contributing SNe and a cosmic selection effect favoring contributions from SNe in a certain mass range for 


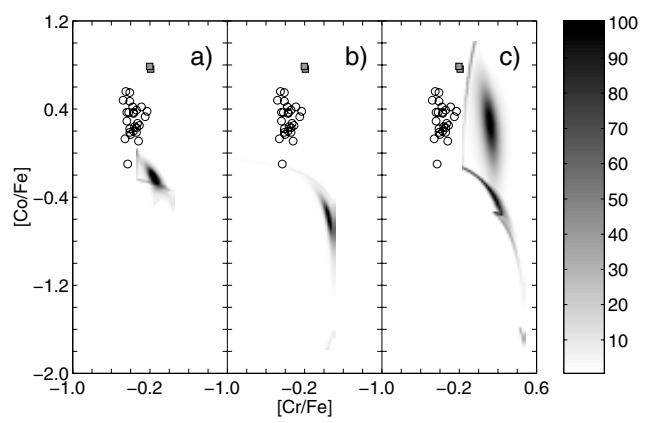

Figure 4. The predicted distributions of stars in the $[\mathrm{Co} / \mathrm{Fe}]-[\mathrm{Cr} / \mathrm{Fe}]$ plane. a) Yields by Woosley \& Weaver (1995). b) Yields by Umeda \& Nomoto (2002). c) Yields by Chieffi \& Limongi (2004). Symbols as in Fig. 3.

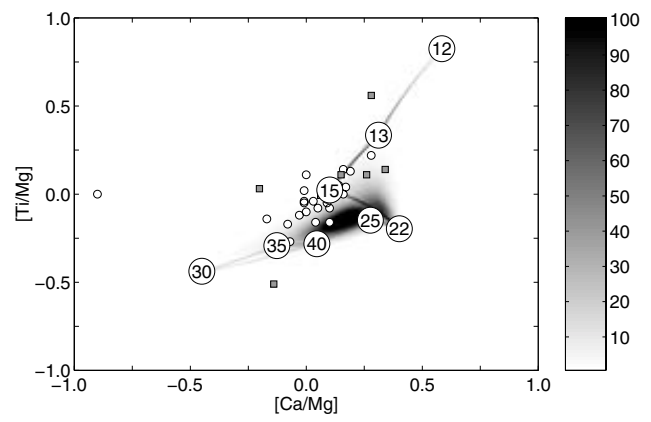

Figure 5. The predicted distributions of stars in the $[\mathrm{Ti} / \mathrm{Mg}]-[\mathrm{Ca} / \mathrm{Mg}]$ plane. The progenitor masses for which SN yields are calculated (Woosley \& Weaver 1995) are encircled. The single-SN origin of the "spurs" are clearly seen (see also Fig. 4). Other symbols as in Fig. 3.

the most metal-poor stars. In the most metal-poor regime the resulting scatter depends crucially on how the $\mathrm{Ca} / \mathrm{Mg}$-yield ratio varies with the $\mathrm{Mg}$-yield. If a large change in $\mathrm{Mg}$ only results in a small change in $\mathrm{Ca} / \mathrm{Mg}$, the scatter will stay small.

When an abundance ratio of two heavy elements is plotted against another ratio of two heavy elements, the uncertainties in the amount of mixing of the SN ejecta with the surrounding medium is nearly eliminated. Most of the remaining uncertainties lies in the stellar yields. As evident from Fig. 4, there are large uncertainties in the calculations of the iron-group yields. None of the yields fit the observations and the diversity between the different predictions is large.

The extended, narrow "spurs" appearing in the calculated distributions in Fig. 4 are not numerical artifacts. They represent the location of stars with major contributions from a single SN, though with different SN masses (see Fig. 5). These features will be called single supernova sequences (SSSs). We judge that observations of at least 200 stars are needed for tracing an SSS, if statistical errors in the abundance observations are about 0.05 dex. If found they will give direct evidence concerning SN yields as well as upper IMFs for the first generation of stars. However, if not found in future accurate surveys, it may indicate the existence of another parameter besides progenitor mass determining the core-collapse SN yields, such as initial angular momentum, unless early element production by SNe with effective mixing of the interstellar gas occurred before any low-mass star was able to form.

\section{Conclusions}

Based on the stochastic model of chemical enrichment presented in Sect. 2, we conclude the following:

- The average number of SNe contributing to the local interstellar abundance increases dramatically with metallicity. At $[\mathrm{Fe} / \mathrm{H}] \simeq-4$, the medium, and the stars formed out of this medium, is mostly enriched by single SNe and a sharp drop in the number of stars with metallicity below $[\mathrm{Fe} / \mathrm{H}]=-4$ is therefore expected. Considering the medium to be well-mixed when the gas is, on average, enriched by $10 \mathrm{SNe}$, the transition from an unmixed to a mixed ISM occurs around $[\mathrm{Fe} / \mathrm{H}]=-3$. At $[\mathrm{Fe} / \mathrm{H}]=-2.5$, the average number of contributing SNe is $>100$ (see Fig. 2). 
- An unmixed ISM does not necessarily imply a large star-to-star scatter in abundance ratios. The cosmic scatter in the most metal-poor regime, for any abundance ratio between two elements $A$ and $B$, depends critically on the variation of the stellar yield ratio $p_{A} / p_{B}$ with the yield of the current metallicity indicator, e.g., element B (see Fig. 3).

- The predicted distributions of stars in diagrams relating different elemental abundances and abundance ratios depend sensitively on the as yet uncertain stellar yields. Observations and predictions agree reasonably well for $\alpha$-elements (see Fig. 3) although the sloping trends are in general more pronounced in the simulations. On the other hand, fore the odd-even elements $\mathrm{Na}$ and $\mathrm{Al}$, the $\mathrm{SN}$ yields tend to give too low abundances and the predicted scatter is smaller than observed (Paper II). The situation for the irongroup elements is even worse. Using our models, the calculated yields are only moderately successful in predicting the relative abundance ratios and their scatter (see Fig. 4). This mismatch between observations and simulations likely reflects the fact that the SN explosion mechanism is not fully understood and the amount of fall-back of ejected material onto the central neutron star/black hole is not known.

- The detection of "spurs", or single supernova sequences (SSSs), in diagrams relating two different abundance ratios would reveal the existence of stars with matter essentially affected by single SNe (see Fig. 5). The chemical abundance ratios in these stars would thus be direct measurements of the corresponding SN yield ratios. Although observationally challenging, requiring samples of several hundred stars with abundance ratios observed to accuracies of 0.05 dex or better, the verification of the existence of SSSs would serve as a strong evidence for a chemically unmixed ISM at the time of formation of the Milky Way Galaxy.

\section{Acknowledgements}

We would like to express our gratitude to François and Monique Spite for their kindness and inspiring way of doing science.

\section{References}

Argast, D., Samland, M., Gerhard, O. E., \& Thielemann, F. K. 2000, A\&\&A 356, 873

Arnone, E., Ryan, S. G., Argast, D., Norris, J. E., \& Beers, T. C. 2005, A\& A 430, 507

Audouze, J. \& Silk, J. 1995, ApJ (Letters) 451, L49

Barklem, P. S., Christlieb, N., Beers, T. C., Hill, V., Holmberg, J., Marsteller, B., Rossi, S., \& Zickgraf, F.-J. 2005, A\&SA in press, [astro-ph/0505050]

Bateman, N. P. \& Larson, R. B. 1993, ApJ 407, 634

Carretta, E., Gratton, R., Cohen, J. G., Beers, T. C., \& Christlieb, N. 2002, AJ 124, 481

Cayrel, R., Depagne, E., Spite, M., Hill, V., Spite, F., François, P., Plez, B., Beers, T., Primas, F., Andersen, J., Barbuy, B., Bonifacio, P., Molaro, P., \& Nordström, B. 2004, A $\& A$ 416, 1117

Chieffi, A. \& Limongi, M. 2004, ApJ 608, 405

Cohen, J. G., Christlieb, N., McWilliam, A., Shectman, S., Thompson, I., Wasserburg, G. J., Ivans, I., Dehn, M., Karlsson, T., \& Melendez, J. 2004, ApJ 612, 1107

Karlsson, T. 2005, $A \mathscr{G} A$ in press, [astro-ph/0504615] (Paper I)

Karlsson, T. \& Gustafsson, B. 2005, A\&A 436, 879 (Paper II)

McWilliam, A., Preston, G. W., Sneden, C., \& Searle, L. 1995, AJ 109, 2757

Nissen, P. E., Gustafsson, B., Edvardsson, B., \& Gilmore, G. 1994, A\&\&A 285, 440

Travaglio, C., Galli, D., \& Burkert, A. 2001, ApJ 547, 217

Tsujimoto, T., Shigeyama, T., \& Yoshii, Y. 1999, ApJ (Letters) 519, L63

Umeda, H. \& Nomoto, K. 2002, ApJ 565, 385

Woosley, S. E. \& Weaver, T. A. 1995, ApJS 101, 181 